\title{
UM MODELO DE MUDANÇA ORGANIZACIONAL CONTÍNUA ATRAVÉS DA GESTÃO DO CONHECIMENTO INTEGRANDO TECNOLOGIA DA INFORMAÇÃO E PESSOAS
}

\section{A MODEL OF CONTINUOUS ORGANIZATIONAL CHANGE THROUGH THE KNOWLEDGE MANAGEMENT INTEGRATING INFORMATION TECHNOLOGY AND PEOPLE}

\author{
Sergio Alexandre de Castro ${ }^{1}$; Edson Walmir Cazarini ${ }^{2}$ \\ ${ }^{1}$ Universidade de São Paulo - São Carlos - Brasil - sergiocastromta@yahoo.com.br \\ ${ }^{2}$ Universidade de São Paulo - São Carlos - Brasil - cazarini@sc.usp.br
}

Recebido para publicação em: 01/03/05 Aceito para publicação em: 15/07/05

\begin{abstract}
Resumo
A busca por vantagem competitiva no mercado globalizado tem influenciado várias mudanças organizacionais nas empresas. Estas mudanças são descontínuas gerando conflitos na organização e perda de competitividade. Este artigo apresenta um modelo de mudança organizacional contínua onde a gestão do conhecimento, através da Metodologia EKD (Enterprise Knowledge Development), integra a tecnologia de informação, os sistemas de informação e as pessoas. Este modelo elimina a descontinuidade das mudanças, pois apresenta uma visão sistêmica para as mudanças e beneficia a criação de vantagens competitivas.
\end{abstract}

Palavras chave: mudança organizacional, tecnologia da informação, gestão do conhecimento.

\section{Introdução}

As empresas brasileiras passaram a perceber o fenômeno da globalização na década de 90 , proporcionado por mudanças de paradigmas onde a informação e processo de negócio passaram a ser mais importante que o produto. Os indicadores de competitividade antes ancorados ao produto, preço e praça, agora estão baseados na qualidade dos processos produtivos, produtividade, habilidade para administrar informações, produzir conhecimento, inovação e satisfação plena dos clientes.

A competição globalizada influencia a forte ligação entre a tecnologia da informação, as mudanças organizacionais e o crescimento da produtividade (CASTELLS, 1999). Neste cenário altamente competitivo e volátil, a criação de vantagens competitivas visa à sobrevivência da organização no mercado. Conforme Coyne (1986), não basta adquirir a vantagem competitiva. É preciso mantê-las. Uma vantagem competitiva é sustentável quando a diferenciação agrega valor ao produto; flexibiliza a entrega do produto; influencia o mercado; encontra e explora brechas no sistema de negócio, brechas legais e brechas na administração ou organização da qualidade. Porter (1999) acrescenta o uso da informação como uma ferramenta essencial para criar vantagem competitiva.

As empresas encontram dificuldades para lidar com a ligação da tecnologia da informação, as mudanças organizacionais e o crescimento da produtividade, sobretudo, pela descontinuidade das 
mudanças. Esta descontinuidade gera imprevisibilidade da sua eficácia e conflitos internos na organização, gerando a perda de competitividade.

Neste contexto, este artigo apresenta um modelo de mudança organizacional contínua focado na gestão conhecimento que integra através da metodologia EKD, a tecnologia de informação, os sistemas de informação e as pessoas no processo de mudança. Então, serão apresentados brevemente conceitos sobre gestão do conhecimento divididos em conceitos, pessoas e metodologia EKD, e também conceitos sobre tecnologia da informação divididos em infraestrutura, sistemas de informação operacionais e sistemas de informação estratégicos. Em seguida será apresentado o modelo de mudança organizacional contínua.

\section{Gestão do Conhecimento}

A abordagem da gestão do conhecimento será divida entre os tópicos: conceitos, pessoas e a metodologia EKD.

\subsection{Conceitos}

Conhecimento é definido por Probst, Raub \& Romhardt (2002) como o conjunto de cognição e habilidades que os indivíduos usam para resolver problemas baseando-se em dados e informações. $\mathrm{O}$ conhecimento é diretamente ligado às pessoas. Afirma ainda que o conhecimento organizacional consiste em ativos de conhecimento individuais e coletivos que a organização pode aplicar na realização de suas tarefas, incluindo também os dados e informações usados na construção do conhecimento individual e organizacional. Defende que o conhecimento deve ser construído de acordo com a relevância para os objetivos da organização.

Wiener (1989) defende que o homem é capaz de aprender por causa da estrutura física do seu cérebro (neurônios complexos) e do seu corpo (sensores). Compara uma comunidade de formigas onde vivem em um sistema fascista que aprisiona a formiga numa função por toda a sua vida. Quem nasce operária sempre será operária, quem nasce rainha sempre será rainha. Isto só ocorre por causa da sua estrutura limitada, tornando-a incapaz de aprender. Já a estrutura do homem permite a aprendizagem e o desenvolvimento. A força da espécie humana está na sua capacidade de adaptação e de aprendizagem.

Parafraseando Wiener pode-se dizer que a organização é capaz de aprender à medida que desenvolve sua estrutura organizacional focando-se na produção do conhecimento pela aquisição e utilização da informação como vantagem competitiva no ambiente globalizado deste novo século.

Muito se fala em mudança de paradigma e mentalidade. Senge (2002) usa o termo grego metanóia que se refere à mudança fundamental da mente. Literalmente trata-se da transcendência da mente como um significado mais profundo de aprendizagem. Apresentam neste contexto de mudança fundamental algumas disciplinas da organização que aprende (Quadro 1).

Define ainda o pensamento sistêmico como a quinta disciplina que integra as outras disciplinas fundindo num só corpo teoria e prática. Dentro deste pensamento a organização não pode ser vista em partes, fracionada em seções e departamentos. Toda a decisão e resolução de problemas isolados acabam resultando em maiores problemas em outros setores da organização e o problema acaba voltando ainda maior de onde ele surgiu. É preciso atacar as causas, as raízes dos problemas e não os sintomas. Para conhecer a causa é necessário olhar o todo. Muitas vezes o efeito de uma decisão só vai ser percebido em longo prazo e em outro setor dificultando o conhecimento da causa. Decisões rápidas e bruscas causam mais danos que benefícios, sendo mais eficazes pequenas atitudes em harmonia com o todo do sistema a grandes decisões isoladas.

Dentro do aspecto do pensamento sistêmico faz-se necessário entender o conceito da realimentação ou feedback. Senge (2002) vê o feedback como qualquer fluxo recíproco de influência. Ele classifica o feedback em três tipos: 
- Feedback de reforço: qualquer movimento que ocorra é amplificado produzindo mais movimentos na mesma direção criando círculos que podem ser "viciosos" ou "virtuosos".

- Feedback de equilíbrio: é autocorreção do sistema que tenta manter a meta ou objetivo. É a busca da estabilidade do sistema.

- Feedback de defasagem: é o intervalo entre as ações e as conseqüências. As defasagens não identificadas podem resultar em instabilidade e colapso, principalmente se prolongadas.

Quadro 1 - Disciplinas da organização que aprende

\begin{tabular}{|l|l|}
\hline \multicolumn{2}{|c|}{ Disciplinas da organização que aprende } \\
\hline Pensamento sistêmico & $\begin{array}{l}\text { Só poderemos entender um sistema contemplando o todo, não uma parte individual do } \\
\text { padrão. As empresas e os outros feitos humanos também são sistemas. Estão igualmente } \\
\text { conectados por fios invisíveis de ações inter-relacionadas, que muitas vezes levam anos } \\
\text { para manifestar seus efeitos umas sobre as outras. Onde é difícil ver o padrão da mudança } \\
\text { como um todo. }\end{array}$ \\
\hline Domínio pessoal & $\begin{array}{l}\text { Disciplina de continuamente esclarecer e aprofundar nossa visão pessoal, de concentrar } \\
\text { nossas energias, de desenvolver paciência e de ver a realidade objetivamente. }\end{array}$ \\
\hline Modelos mentais & $\begin{array}{l}\text { Pressupostos profundamente arraigados, generalizações ou mesmo imagens que } \\
\text { influenciam nossa forma de ver o mundo e de agir. }\end{array}$ \\
\hline $\begin{array}{l}\text { Construção de uma visão } \\
\text { compartilhada }\end{array}$ & $\begin{array}{l}\text { Sua prática envolve as habilidades de descobrir imagens do futuro compartilhadas que } \\
\text { estimulem o compromisso genuíno e envolvimento em lugar da mera aceitação. }\end{array}$ \\
\hline $\begin{array}{l}\text { Aprendizagem em } \\
\text { equipe }\end{array}$ & $\begin{array}{l}\text { Começa pelo diálogo, a capacidade dos membros de deixarem de lado as idéias } \\
\text { preconcebidas e participarem de um verdadeiro "pensar em conjunto". O diálogo envolve } \\
\text { também o reconhecimento dos padrões de interação que dificultam a aprendizagem nas } \\
\text { equipes. Se estes padrões não forem detectados destroem a aprendizagem. Se percebidos e } \\
\text { trabalhados de forma criativa podem acelerar a aprendizagem. }\end{array}$ \\
\hline
\end{tabular}

Fonte: SENGE (2002)

A gestão do conhecimento tem proporcionado à empresa uma visão mais abrangente do papel das pessoas na sua estratégia competitiva, sinalizando a necessidade de melhor aplicação das informações geradas pelos sistemas de informação, criando vantagens competitivas mais consistentes e propiciando às mudanças organizacionais.

\subsection{Pessoas}

Há uma nova natureza do trabalho. Nota-se que hoje, o trabalho é mais cognitivo, mais complexo, mais fluido, mais incerto, mais interconectado e mais invisível. Conseqüentemente, surge um novo perfil do trabalhador exigindo neste mais inteligência, mais diferenciação, mais adaptação, mais responsabilidade, mais relacionamentos e que o trabalhador seja mais voltado para seu crescimento pessoal (BASTOS, 2003).

Neste novo ambiente surge o analista de negócio e o analista de tecnologia, devendo possuir um perfil multidisciplinar. Este analista de negócio e analista de tecnologia tem a responsabilidade de estreitar as relações entre as pessoas da organização com os processos de negócios e a tecnologia.

É desejável em um único profissional a característica do analista de negócio e analista de tecnologia. A gestão do conhecimento deve auxiliar as pessoas na formação deste novo perfil. As cinco disciplinas de Senge (2002), apresentadas no Quadro 1, cooperam com este objetivo.

\subsection{Metodologia EKD}

O EKD (Enterprise Knowledge Development) pode ser uma ferramenta para a construção desta visão sistêmica da organização, pois é uma metodologia que fornece uma forma sistemática e 
controlada de analisar, entender, desenvolver e documentar uma organização e seus componentes, usando a Modelagem Organizacional. Tem por objetivo descrever de maneira clara e não ambígua (PÁDUA, 2001):

- Como a organização funciona atualmente;

- Quais são os requisitos e as razões para a mudança;

- Quais alternativas deveriam ser criadas para encontrar esses requisitos;

- Quais são os critérios e argumentos para avaliação dessas alternativas.

O EKD pode ser visualizado em três níveis: Objetivos organizacionais; processos organizacionais e sistemas de informação. Há também sub-modelos que compõe o EKD: Modelo de Objetivos, Modelo de Regra de Negócio, Modelo de Conceito, Modelo de Processo do Negócio, Modelo de Atores e Recursos, Modelo de Requisitos e Componentes Técnicos. Mais detalhes sobre o EKD e seus modelos em User Guide (BUBENKO et al., 1998).

O EKD pode ser uma ferramenta de integração possibilitando a gestão do conhecimento integrar os sistemas de informação, a tecnologia da informação e as pessoas, facilitando o processo contínuo de mudança organizacional.

\section{Tecnologia da informação}

Uma breve abordagem sobre tecnologia da informação e seus componentes será feita nos tópicos: infra-estrutura, sistemas de informação operacional e sistemas de informação estratégico.

\subsection{Infra-estrutura}

O termo tecnologia da informação é bastante amplo, podendo envolver hardware, software, redes, banco de dados e outras tecnologias de processamento de informação (O'BRIEN, 2003). A tecnologia da informação fornece a infra-estrutura para os sistemas de informação. Como infraestrutura podem-se considerar os computadores e periféricos (hardware), as redes (comunicação) e os bancos de dados.

Uma melhor aplicabilidade dos sistemas de informação tem ocorrido através da evolução dos microcomputadores e periféricos (vídeos, impressoras e recursos multimídia), acompanhado da evolução da comunicação nas redes internas (LANs) e da Internet (WEB), bem como, da evolução dos bancos de dados através do crescimento da capacidade de armazenagem de dados proporcionado pelos novos sistemas gerenciadores de banco de dados (SGBD).

\subsection{Sistemas de informação operacional}

É difícil fazer uma definição axiomática dos sistemas de informação, entretanto, pode-se ressaltar que a sua finalidade está em armazenar e transformar os dados disponíveis na organização em informações úteis para os níveis operacionais, gerenciais e estratégicos. Para o contexto deste artigo os sistemas de informação serão divididos em sistemas de informação operacional e sistemas de informação estratégicos, onde o nível gerencial está englobado no operacional pelo fato de cada vez mais a função gerencial ser necessário para administrar a complexidade dos processos de produção.

Uma informação operacional gerada através de um sistema tem por finalidade permitir que determinadas operações continuem acontecendo dentro do ciclo operacional da empresa (OLIVEIRA, 2004). De acordo com O'Brien (2003), os sistemas operacionais são utilizados para processar transações, controlar processos industriais, apoiar comunicações e colaboração e atualizar banco de dados da empresa. Nesta categoria encontram-se os sistemas de processamento e transações, sistemas de controle de processos e os sistemas colaborativos. 
Um sistema de informação gerencial (SIG) pode ser definido como um método formal para disponibilizar à administração, as informações precisas necessárias para facilitar o processo de tomada de decisão e para dar condições para que as funções de planejamento, controle e operacionais da organização sejam executadas eficazmente (OLIVEIRA, 2000). Esta definição reforça a consideração do SIG como um sistema de informação operacional.

Atualmente as empresas, principalmente as de manufatura, tem utilizado no nível operacional e gerencial o software de gestão ERP. Os sistemas de ERP (Enterprise Resources Planning) são uma evolução do MRP (Material Requirement Planning) que tem a função de calcular a necessidade de materiais para um determinado produto. O MRP evoluiu para o MRP II (Manufacturing Resources Planning) que além da necessidade de materiais calculava também a necessidade dos recursos para a produção de determinado produto. O ERP é basicamente composto de módulos integrados que atendem a necessidades de informação para apoio à tomada de decisão de setores outros que não apenas aqueles ligados à manufatura. Estes módulos são integrados a partir de uma base única de dados buscando a não redundância dos dados (CORRÊA et al, 2001).

\subsection{Sistemas de informação estratégico}

O conceito de cadeia de valores que identifica as várias atividades diferenciadas, do ponto de vista tecnológico e econômico, que a empresa desempenha para executar seu negócio tem sido relevante para o uso da tecnologia de informação na obtenção de vantagem competitiva. Visto que a tecnologia da informação tem influenciado todos os pontos da cadeia de valores, transformando a maneira como as atividades de valor são desempenhadas e a natureza da relação entre elas (PORTER, 1999).

Complementando, os sistemas de informação passam do estágio de sistemas de informação operacional para sistemas de informação estratégico, de modo que estão gerando mais dados à medida que a empresa desempenha suas atividades e está possibilitando a coleta ou a captação de informações que até então não estavam disponíveis. Os novos recursos tecnológicos também abrem espaço para a análise e utilização mais abrangentes dos dados ampliados.

Para O'Brien (2003) os sistemas de informação estratégicos são os que apóiam ou moldam a posição e estratégias competitivas de uma empresa. Neste aspecto, qualquer tipo de sistema de informação pode ser estratégico na perspectiva de colaborar com a organização a obter uma vantagem competitiva, reduzir uma desvantagem competitiva ou alcançar outros objetivos estratégicos.

Os sistemas de apoio à decisão (SAD), - principalmente, os novos componentes: Datewarehouse, OLAP (On-Line Analytical Processing) e Data Mining - e SIE (Sistemas de Informação Executiva) são ferramentas de grande poder para o apoio à estratégia competitiva da empresa.

\section{Modelo de mudança organizacional contínua}

As mudanças organizacionais sempre aconteceram na história da administração, mas os ciclos de mudanças eram mais lentos e percorriam um espaço maior no tempo. Com o advento da tecnologia da informação houve um aumento dos ciclos de mudanças diminuindo seu período de duração. Entretanto, não realizar estas mudanças em tempo hábil pode acarretar a perda de competitividade no mercado cada vez mais veloz.

No ambiente organizacional o termo "mudança" tornou-se uma palavra de ordem, mas muitas empresas não estão conseguindo realizá-las de forma que garantam uma vantagem competitiva e o próprio crescimento da empresa. É necessário o conhecimento organizacional para que as mudanças não se tornem uma armadilha para a organização.

Um modelo de mudança organizacional contínua (Figura 1), onde a gestão do conhecimento integra a tecnologia de informação, os sistemas de informação e as pessoas, são pilares para a organização fazer suas mudanças necessárias. Esta integração tem como suporte a Metodologia 
EKD.

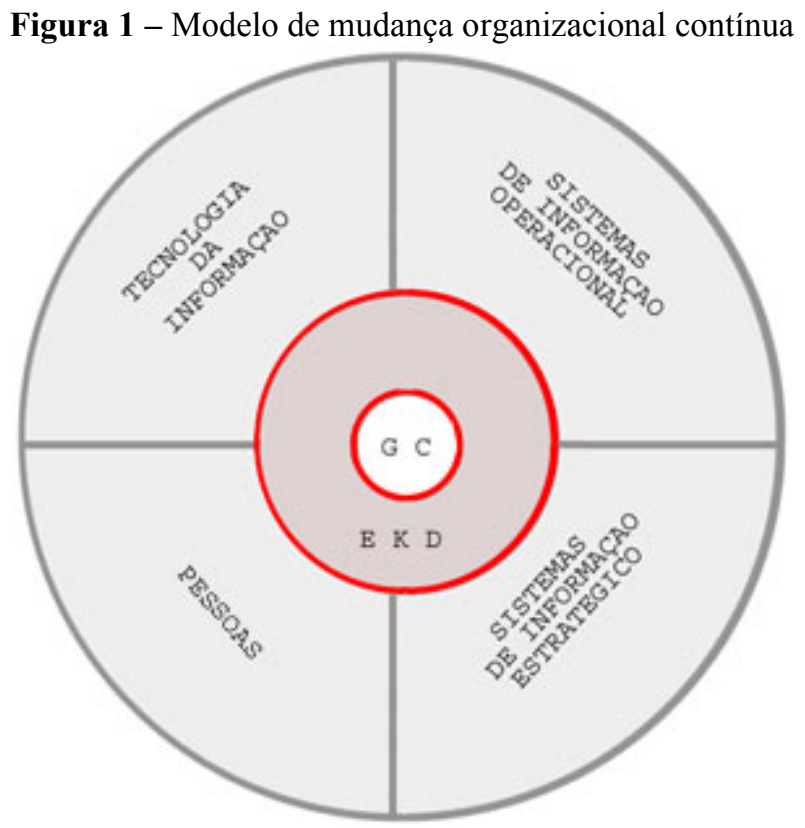

Neste modelo considera-se a organização composta pela tecnologia da informação, sistemas de informação operacional, sistemas de informação estratégico e pessoas. No centro da organização está a gestão do conhecimento (GC) que detêm a capacidade de mudança.

A ligação da gestão do conhecimento com os componentes da organização se dá através dos modelos contidos no EKD. Para cada componente da organização há um modelo correspondente no EKD (Quadro 2).

Quadro 2 - Correspondência dos componentes da Organização e Modelos do EKD.

\begin{tabular}{|l|l|}
\hline \multicolumn{1}{|c|}{ COMPONENTES DA ORGANIZAÇÃO } & \multicolumn{1}{c|}{ EKD } \\
\hline Tecnologia da Informação & Modelo de Requisitos e Componentes Técnicos \\
\hline Sistemas de Informação Operacional & Modelo de Conceitos e Modelo de Processos de Negócio \\
\hline Sistemas de Informação Estratégico & Modelo de Objetivos e Modelo de Regras de Negócio \\
\hline Pessoas & Modelo de Atores e Recursos \\
\hline
\end{tabular}

Os processos de mudanças não são isolados neste modelo. Precisam ocorrer de forma sistêmica e simultânea, verificando a influência das mudanças em cada componente da organização.

Como exemplo pode-se considerar uma mudança nos sistemas de informação operacional em razão da implementação de um sistema de gestão integrada (ERP). Segundo Lima et al (2003), a adoção de um sistema ERP implica em um processo de transformação organizacional, onde o redesenho de processos e mudanças organizacionais são essenciais para atingir os objetivos propostos por um sistema de informação. Defende as estruturas baseadas em processos em lugar da estrutura organizacional hierarquizada. Nesta mesma linha, Stamford (2003), afirma que as mudanças envolvem estrutura, operação, estratégia e até mesmo a cultura da empresa.

Neste exemplo, a gestão do conhecimento expande os processos de mudança para toda a organização explicitando as mudanças no Modelo de Conceitos e Modelo de Processos de Negócio. Uma vez demonstrado as mudanças no sistema de informação operacional, verifica-se sua influência na tecnologia da informação confrontando as mudanças com o Modelo de Requisitos e Componentes.

Sendo encontrada alguma inconformidade entre os modelos, o processo de mudança deve ser realizado também na tecnologia da informação, por exemplo, precisa-se mudar o sistema 
gerenciador de banco de dados ou a arquitetura da Rede. Esta mudança na tecnologia da informação deve ser relatada no Modelo de Requisitos e Componentes Técnicos e retornar para o processo de validação.

Confronta-se, então, o Modelo de Conceitos, Modelo de Processos de Negócio e Modelo de Requisitos e Componentes Técnicos com o Modelo de Objetivos e Modelo de Regras de Negócio. Exemplificando, percebe-se uma oportunidade de negócio com estas mudanças no sistema de informação operacional e na tecnologia da informação. Definem-se novas regras de negócio e objetivos novos são traçados. Esta oportunidade é transferida para a gestão do conhecimento através da construção de um novo Modelo de Regras de Negócio e Modelo de Objetivos.

Fechando o processo, confrontam-se as mudanças no sistema de informação operacional, sistema de informação estratégico e tecnologia da informação com o componente "pessoas" através do Modelo de Atores e Recursos. Por exemplo, percebe-se a necessidade de um analista de negócios e analista de tecnologia. O processo de mudança ocorre neste componente através da formação ou a contratação deste profissional.

Concluindo, a gestão do conhecimento integra o processo de mudança em todos os componentes da organização através do EKD, sobretudo, para que a mudança organizacional em um componente não venha prejudicar os demais e gerar um problema futuro. Tenta-se com este modelo realizar as mudanças necessárias de forma sistêmica, criando vantagens competitivas ou eliminando desvantagens competitivas.

\title{
5. Considerações finais
}

O modelo proposto elimina a descontinuidade das mudanças, pois as mudanças passam a ser constante. Os problemas, independente de onde eles estejam situados, são discutidos e resolvidos em toda a organização. As mudanças deixam de ser arbitrárias, e passam a ser consentidas, tendo o comprometimento de todos.

A cada processo de mudança na organização, a gestão do conhecimento tende a crescer desenvolvendo um potencial para transformar os riscos e incertezas gerados no mercado globalizado em vantagens competitivas para o fortalecimento e crescimento da organização.

\begin{abstract}
The search for competitive advantage in the globalized market has influenced some organization changes in the companies. These changes are discontinous generating conflicts in the organization and loss of competitiveness. This article presents a model of continuous organizational change where the knowledge management, through Methodology EKD (Enterprise Knowledge Development), integrates the information technology, the systems of information and the people. This model eliminates the discontinuity of the changes, therefore it presents a systemic view for the changes and benefits the creation of competitive advantages.
\end{abstract}

Key words: Change Organizational, Information Technology, Knowledge Management.

\section{Referências}

BASTOS, A.V.B. Aprendizagem \& competência organizacional: os desafios da contemporaneidade. In: SIMPEP, 10., Bauru. Anais... Bauru, 2003.

BUBENKO, J. R. et al. EKD user guide. Stockholm: Royal Institute of Technology. 1998.

CASTELLS, M. A sociedade em rede: a era da informação: economia, sociedade e cultura. São Paulo: Paz e Terra. 1999. v. 1. 
CORREAA, H. L.; GIANESI, I. G. N.; CAON, M. Planejamento, programação e controle da produção: MPR II/ERP: conceitos, uso e implantação. São Paulo: Atlas, 2001.

COYNE, K. P. Sustainable Competitive Advantage: What Is Is, What It Isn't. Business Horizons, p.54-61, Jan./Febr. 1986.

LIMA, A.D.A. et al. Implantação de pacotes de gestão empresarial em médias empresas. Disponível em: http://www.kmpress.com.br. Acesso em: 18 dez. 2003.

O’BRIEN, J. A. Sistemas de informação e as decisões gerenciais na era da internet. São Paulo: Saraiva, 2003.

OLIVEIRA, J. F. Sistemas de Informação: um enfoque gerencial inserido no contexto empresarial e tecnoloógico. São Paulo: Érica, 2000.

Sistemas de Informação versus tecnologia da informação: um impasse empresarial. São Paulo: Érica, 2004.

PÁDUA, S. I. D. Investigação do processo de desenvolvimento de software a partir da modelagem organizacional, enfatizando regras de negócio. Dissertação (Mestrado). Escola de Engenharia de São Carlos - USP, 2001.

PORTER, M. E. Competição= On competition: estratégias competitivas essenciais. Rio de Janeiro: Campus, 1999.

PROBST, G.; RAUB, S.; ROMHARDT, K. Gestão do conhecimento: os elementos construtivos do sucesso. Porto Alegre: Bookman, 2002.

SENGE, P. M. A quinta disciplina: arte e prática da organização que aprende. São Paulo: Best Seller, 2002.

STAMFORD, P. P. ERP: prepare-se para esta mudança. Artigo publicado pela KMPess. Disponível em: http://www.kmpress.com.br. Acesso em: 18 dez. 2003.

WIENER, N. Cibernética e sociedade: o uso humano de seres humanos. São Paulo: Cultrix, 1989. 